\title{
Hydromagnetic Wave in an Inhomogeneous, Cylindrical Plasma
}

\author{
C. K. McLane and T. Tsukishima ${ }^{1}$
}

\author{
Contribution From Institute for Basic Standards, National Bureau of Standards, Washington, D.C.
}

(Received October 23, 1964)

\begin{abstract}
A transverse, comparatively low-frequency standing wave has been identified on the plasma column of a magnetically confined argon arc. The frequency of this wave, under some conditions, obeys a dispersion relation given by Weinstock for a generalized hydromagnetic wave in an inhomogeneous, rotating plasma. The wave frequency, which may be higher than the ion gyrofrequency, is closely coupled with the fluid rotation velocity of the plasma. When the differences between these passes the ion gyrofrequency, transition to another, as yet unidentified, mode takes place.
\end{abstract}

\section{Introduction}

Abundant experimental observations on hydromagnetic waves in homogeneous, quiescent laboratory plasmas exist [see, for example, Jephcott, 1959; Nagao and Sato, 1960; Wilcox, DeSilva, and Cooper, 1961; Jephcott and Malein, 1964]. It is the purpose of this paper to present experimental observations on a lowfrequency hydromagnetic wave in an inhomogeneous, rotating plasma. Such waves are qualitatively different from the well-known homogeneous plasma waves in several important respects. First, the frequency of this wave is closely coupled, but not equal, to the frequency of the fluid rotation of the plasma. Second, the frequency of this wave, even when larger than the ion gyrofrequency, may be much less than that of an Alfvén wave for the same $\mathrm{k}$ in a homogeneous plasma (if an Alfvén wave could exist under these conditions). Third, the frequency is much less dependent on the density of the plasma than on the magnetic field. The wave will be identified by comparing its properties with those predicted by a dispersion equation derived for a theoretical model of the rotating cylindrical plasma by Weinstock [1964].

The plasma used in this investigation is that of the magnetically confined, low pressure argon arc [McLane, Takeda, Thomas, and Thompson, 1965]. For the operation of this arc, three electrodes alined with a uniform ( \pm 3 percent) magnetic field are utilized. Between the first, which is a $1 / 8$ in. diam. thoriated tungsten cathode, and the second, which is a tungsten nozzle with a $1 / 8 \mathrm{in}$. bore, a $1 / 2$ in., $80 \mathrm{~A}$ arc operates in argon at about 1 torr pressure. This arc acts as a virtual cathode for the main discharge, which

${ }^{1}$ On leave from the Nagoya University, Nagoya, Japan. operates at $8 \mathrm{~A}$ (for this work) in argon at $10^{-3}$ torr between the nozzle and the third electrode, which is a hollow anode consisting of a stack of spaced tantalum washers. The length of the main discharge is variable, is referred to as the arc length, and denoted by $L$ in what follows. Further experimental details on the discharge and on the method of measuring electron density and temperature distributions may be found in the reference cited above. ${ }^{2}$

The plasma of the main discharge is a very stable, cylindrical ribbon whose peak (axial) density is from $10^{19}$ to $10^{20} \mathrm{~m}^{-3}$ and whose half density radius is from 0.4 to $0.5 \mathrm{~cm}$. The axial temperature is from 1.5 to $3 \mathrm{eV}$ and the half temperature radius is from 0.5 to $0.9 \mathrm{~cm}$. At the densities and temperatures quoted, the collision frequencies for momentum exchange may be compared as follows: the electron-ion collision frequency $\left(10^{8}-10^{9} \mathrm{sec}^{-1}\right)$ much exceeds the electronneutral collision frequency $\left(10^{3}-10^{4} \mathrm{sec}^{-1}\right)$; the ionneutral collisions $\left(10^{5} \mathrm{sec}^{-1}\right)$ dominate the ion-electron collisions $\left(10^{3}-10^{4} \mathrm{sec}^{-1}\right)$. The degree of ionization is from about 25 percent to over 75 percent. The ion temperature has been estimated by the same method used on a similar plasma by Lidsky et al. [1962] to be approximately $1 \mathrm{eV}$ (error may be a factor of two either way).

What concerns us here is that this plasma, under nearly all conditions of its existence, is in continuous oscillation. [McLane, Thomas, and Thompson, 1963]. The most commonly encountered modes are transverse, and it is our purpose to demonstrate that one of these modes is, in all probability, associated with a generalized hydromagnetic wave.

${ }^{2}$ It should be noted that the density and temperature distribution in the plasma, and the oscillation modes which are excited, will be influenced by the bore size and bore length of the nozzle electrode, but not appreciably by the current in the arc operating between cathode and nozzle. 


\section{Experimental Measurement of Oscillation and Rotation Frequencies}

Because it does not disturb the plasma, the best method of observing the oscillations is to use a lens to image the plasma column on a thin metal screen and place a photomultiplier behind a small hole in the screen at the place where the oscillation is to be observed. Similar results can be obtained with, in most cases, remarkably little disturbance to the plasma, by placing a small Langmuir probe $(1 / 4 \mathrm{~mm}$ diameter $\times 1 / 2$ to $1 \mathrm{~mm}$ long) near it. The oscillations can then be observed in either the ion saturation current, or, better, in the floating potential. The photoelectric method was used for most of this work.

Transverse waves of odd azimuthal wave number ( $m$ in the wave phase factor $e^{i(\omega t+k z+m \theta)}$ ) are easily distinguished by noting that the oscillating component of the light seen by the photomultiplier will suffer a phase reversal as the point of observation crosses the axis of the plasma column. For odd $m$, the amplitude of the oscillation on axis will be zero. Conversely, if a maximum amplitude is found on the axis, the wave is either longitudinal, or transverse with even $m$. These conclusions are valid if the perturbations, when represented as functions of the radius, show no sign change. This is the case for the oscillation discussed in this paper.

A survey of the most important and reproducible odd $m$ modes seen in the magnetically confined arc plasma in magnetic fields from 0.05 to $0.73 \mathrm{~T}$ ( 1 tesla equals $10^{4}$ gauss) is presented in figure 1 . For convenience in our discussion, these modes will be referred to as I, II, III, and IV as shown in the figure. Mode II occurs at a frequency generally above the ion gyrofrequency, and it is this mode that we shall consider in some detail in this paper. Modes I, III, and IV appear in an intermediate field, but I and IV become strongest and

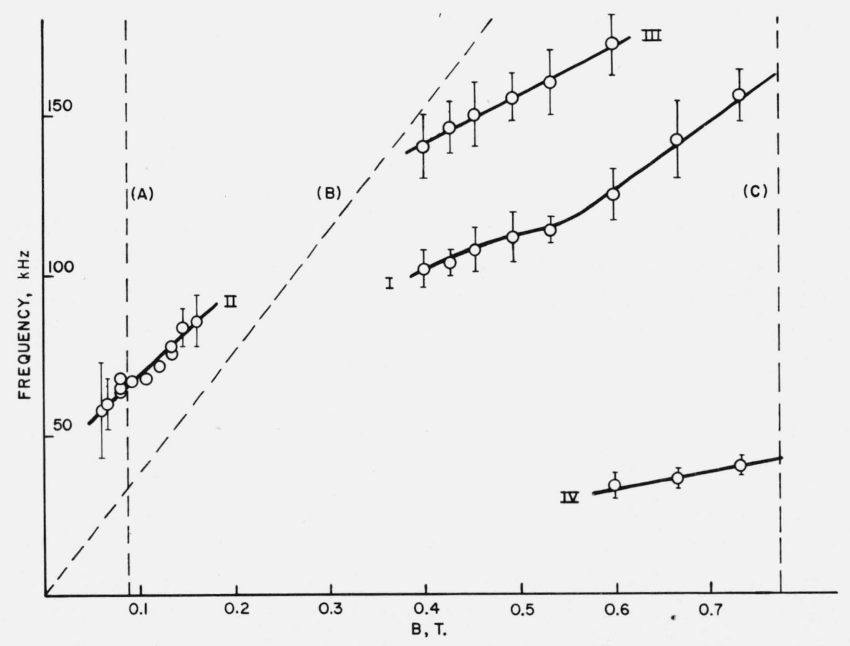

FIGURE 1. Transverse, odd $\mathrm{m}$ oscillation modes of magnetically confined arc plasma.

Dashed lines: (A) locates the field at which the ion gyroradius is about three times the plasma radius; (B) locates the ion gyrofrequency; and (C) locates the field at which the ion plasma radius; (B) locates the ion gyrofrequency;
gyroradius is about one-third the plasma radius. sharpest when the conditions for the ideal hydromagnetic fluid begin to be satisfied.

In addition to these odd $m$ modes a persistent even $m$ or longitudinal mode is seen at 15 to $20 \mathrm{kHz}$ over a wide range of fields. Its frequency and field independence suggest that it may be the ion acoustic wave seen in a somewhat similar plasma by Alexeff and Neidigh $[1961,1963]$. There are also others which are neither so reproducible nor distinct as the foregoing in the region where the ion gyroradius is of the same order of magnitude as the plasma column radius.

TABLE 1. Oscillation and rotation frequencies of plasma column ${ }^{\text {a }}$

\begin{tabular}{|c|c|c|c|c|c|c|c|c|c|c|c|}
\hline $\begin{array}{l}\pi / L \\
\mathbf{M}^{-1}\end{array}$ & $\begin{array}{l}B \\
T\end{array}$ & $\omega$ & $\begin{array}{c}W \\
\text { obs. }\end{array}$ & $\begin{array}{c}W \\
\text { calc. }\end{array}$ & $\omega_{b i}$ & $\begin{array}{l}W_{c} \\
\text { calc. }\end{array}$ & $\frac{k^{2} v_{A}^{2}}{\omega_{b i}}$ & $\begin{array}{l}T_{e o} \\
\mathrm{e} V\end{array}$ & $\begin{array}{c}a_{T} \\
\mathrm{~cm}^{-2}\end{array}$ & $\begin{array}{c}n_{e o} \times 10^{-19} \\
\mathrm{~m}^{-3}\end{array}$ & $\begin{array}{c}a_{n} \\
\mathrm{~cm}^{-2}\end{array}$ \\
\hline 6.9 & 0.106 & 0.46 & 0.07 & 0.39 & 0.256 & 1.42 & 0.40 & 1.8 & 1.23 & 6.2 & 2.94 \\
\hline 20.7 & .133 & .94 & .23 & .70 & .321 & 2.59 & 5.8 & 2.2 & 3.0 & 4.8 & 5.1 \\
\hline 6.9 & .186 & b. .44 & .25 & .30 & .449 & 0.97 & 0.39 & 1.7 & 0.86 & 11.2 & 4.42 \\
\hline 7.7 & .186 & c. 41 & .20 & .21 & .449 & 1.35 & .78 & 1.6 & 2.27 & 6.9 & 5.55 \\
\hline 8.8 & .186 & .49 & .34 & .20 & .449 & 1.13 & 1.72 & 1.55 & 2.20 & 4.1 & 4.55 \\
\hline 10.6 & .186 & .55 & .32 & .22 & .449 & 1.40 & 3.15 & 1.8 & 2.08 & 3.25 & 5.1 \\
\hline 12.4 & .186 & .62 & .28 & .30 & .449 & 1.42 & 2.88 & 1.8 & 2.74 & 4.9 & 4.54 \\
\hline 15.5 & .186 & .87 & .40 & .5 & .449 & 1.42 & 10.2 & 2.0 & 3.33 & 2.15 & 3.22 \\
\hline 20.7 & .186 & 1.10 & .28 & .7 & .449 & 1.58 & 11.8 & 2.2 & 3.06 & 3.3 & 3.57 \\
\hline
\end{tabular}

a All angular velocities in this table $(\omega, W$, etc.) are given in megaradians per second. ${ }^{b}$ Mode II just begins to appear at this value of $\pi / L$ as a band of noise extending from 0.22 to $0.66 \mathrm{Mrad} / \mathrm{sec} . \omega$ is taken to be the middle of this band.

${ }^{c}$ A second frequency, apparently of another mode, appeared at $0.58 \mathrm{Mrad} / \mathrm{sec}$. It was ignored.

The mode II oscillation behaves like a standing wave, in that its frequency is sensitively dependent on the length of the discharge between the nozzle and anode. (See, for example, columns 1 and 3 of table 1.) This was also checked by measuring the longitudinal variation of the amplitude of the oscillation. The amplitude was measured as the rms value, $I^{\prime}$, of the oscillating component of the light intensity. $I^{\prime}$, however, contains a dependence on radius and longitudinal position due to inhomogeneity. In order approximately to eliminate this $R$ and $z$ dependence, we choose to express the amplitude as an equivalent transverse displacement of the plasma column, $\Delta R$, defined by:

$$
\Delta R=\frac{I^{\prime}}{(\partial \bar{I} / \partial R)}
$$

$\Delta R$ is found experimentally to be nearly independent of the radius, thus providing evidence that the perturbation quantities as functions of radius for mode II do not show a sign change. The quantity, $\Delta R$, is shown in figure $2 \mathrm{~A}$ as a function of the $z$-coordinate. The shape of this plot indicates that mode II is a standing wave, and that the wavelength is approximately twice the column length.

Some idea of the absolute magnitude of the amplitude can be obtained by comparing the size of $\Delta R$, as shown in figure $2 \mathrm{~A}$ with the width of the plasma column at half intensity, which is shown in figure $2 \mathrm{C}$ in the same length units used in figure $2 \mathrm{~A} . \Delta R$ is thus found even at its maximum, near the midpoint of the column, to be less than 10 percent of the column width. 

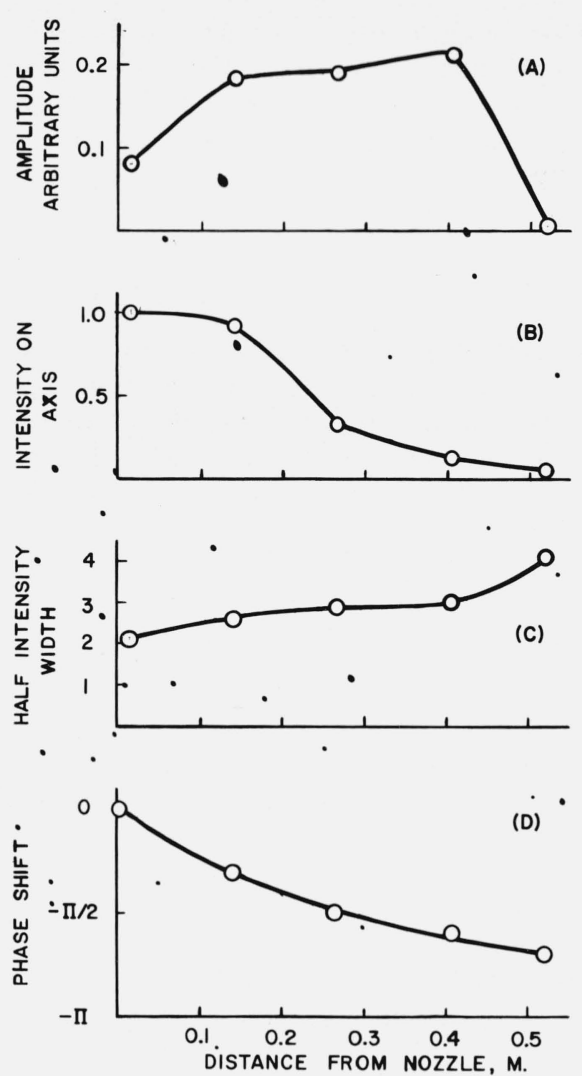

FIGURE 2. z-dependence of some properties of the magnetically confined arc plasma.

(A). Amplitude of the mode II oscillation, measured in arbitrary units.

(B). Light intensity on the plasma column axis relative to that at the cathode end

(C). Half-intensity full width of the plasma column. Units are the same as in figure 2A (Three divisions on this arbitrary scale represent approximately $4 \mathrm{~mm}$ in half intensity full width of the plasma column as seen in the light of the second argon spectrum.)

(D). Phase angle of the oscillation. This is the same as the angle of the helix described by the perturbation for this case, since $|m|=1$.

The departure of the amplitude in figure $2 \mathrm{~A}$ from sinusoidal shape is not at all surprising in view of the longitudinal inhomogeneity of the plasma column. This is dramatized in figure $2 \mathrm{~B}$ which shows the luminous intensity along the column. The decrease in density and temperature which is responsible for this is characteristic of $\mathrm{d}$-c discharges of this kind.

Figure 2D shows that there is a twist in the standing wave of the mode II oscillation. If the phase of the traveling wave is given by $e^{i(\omega t+k z+m \theta)}$, one would expect that of the reflected wave to be $e^{i(\omega t-k z+m \theta)}$. Composition of such waves gives $\cos k z e^{i(\omega t+m \theta)}$, which has a $z$-dependence of the amplitude similar to what is observed, and a perturbation traveling uniformly in the $\theta$-direction, as is observed, but no helicity. We have not examined the details of boundary conditions for reflection of this wave, but have concluded that a small attenuation of the wave (consistent with the observed width of the resonance) could cause the helicity seen in figure 2D.

That mode II consists of a purely odd $m$ transverse wave is seen from figure 3 , which shows the amplitude of the oscillating component of the light output as the photomultiplier traverses the plasma column. The

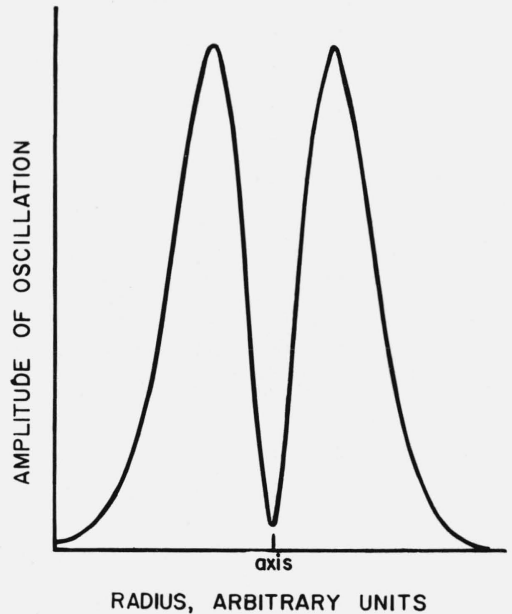

FIGURE 3. Variation of amplitude of mode II oscillation across plasma column.

Reference mark on axis of abscissae locates column axis.

sharp dip, located at the column axis, precludes the possibility of even $m$ transverse or longitudinal mode contribution to this oscillation.

Taking the direction of the magnetic field to be positive, the perturbation is found to rotate in the positive $\theta$-direction. If the phase factor of the wave is $e^{i(\omega t+k z+m \theta)}, m$ must be negative. Comparison of phases of signals from photomultipliers displaced by angles $\pi / 2$ around the column, and of signals from a pair of Langmuir probes which subtended an angle of 20 deg. at the column axis showed that $m=-1$.

Having thus characterized the mode II oscillation for one length $(0.54 \mathrm{~m})$ and one field $(0.133 \mathrm{~T})$ it remained to explore it over as wide a range of physical conditions as was practicable. Mode II as seen under the conditions of figure 1, however, does not represent a good starting point. As seen in figure $2 \mathrm{~B}$, an attempt to vary conditions by changing discharge length may be expected to cause the longitudinal inhomogeneity, as well as $k$, to vary. The result is a dependence of frequency on length that is too complicated to interpret. At the same time, an increase in magnetic field (which, by decreasing transverse diffusion, improves the longitudinal homogeneity) is limited, because mode II becomes noisy and is lost as its frequency approaches the ion gyrofrequency. In order to give maximum longitudinal homogeneity, the approach chosen was to increase $B$ just beyond the range of mode II, then decrease length until mode II reappeared, and scan it as a function of arc length. The data obtained are given in table 1, which also includes two typical examples of the data obtained at lower fields.

It was also necessary to determine the angular velocity of fluid rotation of the plasma (which occurs under the combined influence of the radial components of the electric field and the pressure gradient). This was measured via the Doppler shift of several spectral lines of AII $(3520.00,3521.27,3535.33,3545.58$, 
3545.84, and 3548.51 ̊.) Two spectra, one from a measured distance either side of the plasma column axis, were photographed side by side in the third order of a $1.5 \mathrm{~m}$. Czerny-Turner spectrograph with a 1200 line/mm grating (Applied Research Laboratories Quantograph, Model QSMP. The dispersion of this instrument is $1.52 \AA / \mathrm{mm}$ in the third order.) The photographic plate was not moved-the spectra were positioned by masking the slit. After removing the mask, a mercury spectrum was photographed over the two argon spectra for reference. Three lines of this reference spectrum $(2652.04,2653.68$, and $2655.12 \AA)$, when photographed in fourth order, were conveniently located for measuring the shifts between the members of each pair of argon spectra. The shifts, which varied from 1.7 to $9.3 \mu$ (each value a mean of six determinations) were measured with a photoelectric scanning comparator which could reliably reproduce settings on a spectral line to $1 \mu$. The angular velocities, computed from the measured Doppler shifts and the radius at which the spectra were taken, are recorded in column 4 of table 1 .

\section{Density and Temperature Distributions}

The density distributions were measured by means of the ion saturation current to a Langmuir probe, these having been shown to be reliable for this discharge in previous work [McLane, Takeda, Thomas, and Thompson, 1965]. For convenience in using the densities in the calculations to follow, they were graphically fit with Gaussian profiles from the axis out to $5 \mathrm{~mm}$ radius. The constants for this fit:

$$
n_{e}=n_{e 0} \exp \left(-a_{n} R^{2}\right)
$$

are recorded in columns 11 and 12 of table 1 . The scatter of the points about the Gaussian was essentially random and varied from \pm 3.5 percent for the longer arc to \pm 16 percent for the shortest ones, for which the density gradient was larger and the positioning error of the probe was more serious.

The electron temperatures were taken from the logarithmic slope of the electron collection portion of the Langmuir probe characteristics. These, too, were fit by Gaussian profiles, and the constants from

$$
T_{e}=T_{e o} \exp \left(-a_{T} R^{2}\right)
$$

are recorded in columns 9 and 10 of table 1 . For the long arcs, the scatter of points about the Gaussians is \pm 5 percent if we omit several points near the axis, which appear to be too high (possibly due to overheating of the probe in this very flat temperature profile). This seems justified for the longest arcs, $\left(\pi / L=5.8 \mathrm{~m}^{-1}\right)$ because the conductance of the plasma having the profile obtained from the probe data by neglecting these high points agrees to within 10 percent $\left(6 \%\right.$ for $\left.T_{e}\right)$ with that measured with a small radio frequency current superposed on the $8 \mathrm{~A}$ d-c arc operating current. (The method of measurement will be reported elsewhere.) For the short arcs, there appears to be no problem in the probe measurements other than positional inaccuracy and the scatter of all points is \pm 8.5 percent. This is substantiated in one case $\left(\pi / L=15.5 \mathrm{~m}^{-1}, B=0.186 \mathrm{~T}\right)$ by the $\mathrm{RF}$ measurement, which gave a conductance within 15 percent $\left(10 \%\right.$ for $\left.T_{e}\right)$ of that calculated from the probe data.

\section{Comparison of Experiment With Theory}

For an inhomogeneous, rotating, cylindrical plasma column, Weinstock [1964] has derived the following dispersion equation for a generalized hydromagnetic wave, including the effect of compressibility and finite ion gyrofrequency:

$$
\begin{gathered}
\omega=(1-|m|) W-|m| W_{c} / 2-k^{2} v_{A}^{2} / \omega_{b i} \\
+\left[\left(W+|m| W_{c} / 2+k^{2} v_{A}^{2} / \omega_{b i}\right)^{2}+2 k^{2} v_{A}{ }^{2}\right]^{1 / 2}
\end{gathered}
$$

wherein:

$\omega$ is the angular frequency of the wave,

$W$ is the angular frequency of fluid rotation of the plasma,

$W_{c}$ is the angular frequency associated with the diamagnetic current,

$k$ is the wave number,

$v_{A}$ is the Alfvén velocity, and

$\omega_{b i}$ is the angular ion gyrofrequency.

$W, W_{c}$ and $v_{A}$ are to be determined at the axis of the column. $W_{c}$ is given by

$$
W_{c}=-\frac{\nabla p_{e}}{\operatorname{Ben}_{e} R}-\frac{E}{R B}
$$

wherein $p_{e}$ is the electron pressure, $E$ is the electric field, and the other symbols have their previously defined, or usual, significance. The Alfvén speed is, of course, $B /(\mu \rho)^{1 / 2}$. In writing (3) and defining $v_{A}$, small correction terms given by Weinstock io account for the effect of the arc current are omitted. These are negligible for the plasma used in this work.

It is useful to notice that if the terms $W$, and $W_{c}$, which are introduced by the inhomogeneity of the plasma, are set equal to zero, (3) reduces to:

$$
\omega=-k^{2} v_{A}^{2} / \omega_{b i}+\left[\left(k^{2} v_{A}^{2} / \omega_{b i}\right)^{2}+2 k^{2} v_{A}^{2}\right]^{1 / 2}
$$

Aside from factors of 2 , which are introduced into (3), and hence, (5), by the finite cylindrical geometry of the plasma, (5) is equivalent to the dispersion equation for the slow hydromagnetic wave in the infinite homogeneous plasma for frequencies much lower than the ion plasma frequency. Further, if $\omega_{b i}>k v_{A},(5)$ reduces to

$$
\omega=2^{1 / 2} k v_{A},
$$

which, aside from the $2^{1 / 2}$ resulting from geometry, is the Alfvén wave.

In (4), the second term requires a knowledge of the gradient of the plasma potential. Since this is of the 
order of the ion temperature divided by the plasma diameter and the electron temperature is appreciable, it is difficult if not impossible to measure. However, on the basis of the estimated ion temperature, this term should be small - of the order of 10-15 percent of $W_{c}$ under all conditions reported in the table. It was therefore omitted in calculating the values given in column 7 from the density and temperature profiles.

It is then straightforward to solve (3) for $W$, and compute the values given in column 5 of the table from the data presented in the other columns. Comparison of the measured and calculated fluid rotation velocities shows that:

(1) The agreement is poor for the data given in the first two and the last lines of the table.

(2) In the other cases, viz, $\pi / L$ values of 6.9 to 15.5 $\mathrm{m}^{-1}$ in a magnetic field of $0.186 \mathrm{~T}$, the agreement appears to be good.

The effect of the errors estimated in the previous section for the density and temperature measurements is easily taken into account by solving (3) for $W$ and differentiating it with respect to appropriately chosen variables. It is found that $W$ is quite insensitive to $W_{c}$-and therefore its value, as calculated from $\omega$ and other experimental quantities, is insensitive to experimental errors in the determination of the density and temperature profiles in all cases recorded in table 1. Further support to this conclusion is provided by figure 4 , which shows that variation of pressure and discharge current over rather wide ranges (which have a strong effect on density and temperature distribution) have a much smaller effect on the frequency of the mode II oscillation than does variation in magnetic field (fig. 1).

The major contributors to the errors in $W$ are the wave number, $k$, the Alfvén speed, $v_{A}$, and especially the frequency, $\omega$, whose absolute error appears almost directly in the calculated $W$. If no allowance is made for the frequency error, since $\omega$ and $W$ (observed) were measured simultaneously, allowances are made for density and temperature errors of the order estimated in the previous section, and an error of 10 percent is assumed in setting $k$ equal to $\pi / L$, we can come to the following conclusions:

(1) Differences between $W$ obs and $W$ calc of the order seen in the first two lines and the last line of the table can probably not be accounted for by any reasonable estimate of experimental errors.

(2) Differences of the order seen in all the rest of the table (lines 3 through 8 ) can readily be accounted for in terms of experimental errors.

The data in the first line of the table were taken at a comparatively low field and long length. The effect of the longitudinal inhomogeneity shown in figure $2 \mathrm{~B}$ is therefore expected to be largest in this case. It has already been stated that the $k$-dependence of the experimental frequencies is complicated here. In addition, we cannot be sure that the quantities $W_{c}, k$, and $v_{A}$, based on measurements made in the midplane of the arc column, are the proper mean values to use in order to take account of the longitudinal inhomogeneity. The calculated values of $W$ may therefore be incorrect.

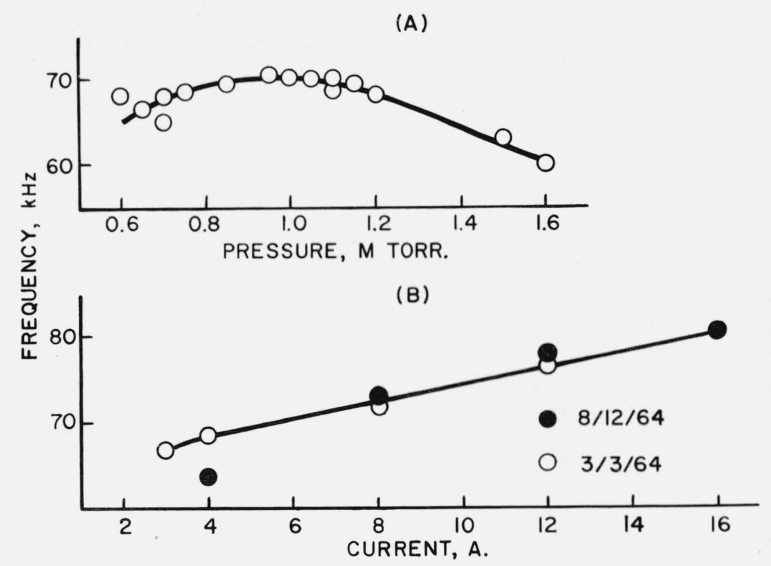

Figure 4. (A). Dependence of mode II frequency on pressure (Discharge current, 8A; magnetic field, $0.106 \mathrm{~T}$; length, $0.54 \mathrm{~m}$ ).

(B). Dependence of mode II frequency on discharge current (pressure, $0.95 \mathrm{~m}$ torr.; magnetic field, $0.106 \mathrm{~T}$; length, $0.54 \mathrm{~m}$ ).

The results for the largest values of $\pi / L$ (lines 2 and 9) are best viewed in terms of the limiting form of (3) for large $k^{2} v_{A}^{2} / \omega_{b i}$

$$
\omega^{\prime} \equiv \omega-(2-|m|) W \approx \omega_{b i}-\frac{1}{2} \frac{\omega_{b i}^{3}}{k^{2} v_{A}^{2}}
$$

For $|m|=1$, this sets an upper limit on the frequency measured relative to the rotating plasma which is equal to the ion gyrofrequency. In the sequence of lines 3 to 9 of the table, it is clear that when $\omega^{\prime}$ clearly surpasses $\omega_{b i}$ with increasing $k^{2} v_{A}^{2} / \omega_{b i}$, the measured and calculated values of $W$ no longer agree. This is not surprising, for this is just the condition under which the wave described by (3) can no longer existtransition to another $k$ or $m$ value will not, in general, lead to a possible solution if $\omega^{\prime}>\omega_{b i}$. While the plasma nevertheless appears to continue oscillating with a similar frequency in a transverse mode above this resonance, the detailed study of the structure of the wave which would be necessary to identify the mode is difficult for such short discharges, and has not been done.

\section{Conclusion}

A transverse, standing wave oscillation has been seen on the plasma column of a magnetically confined arc. Over a two to one range of wave numbers and frequencies, it obeys a dispersion equation applicable to a generalized hydromagnetic wave in a rotating, inhomogeneous, cylindrical plasma. The agreement is lost when the frequency relative to the rotating plasma surpasses $\omega_{b i}$, and similar waves in which the fluid rotation velocity is abnormally small occur. These have not been identified.

The authors express their gratitude to J. Weinstock for many very helpful discussions of his theoretical 
work. Particular thanks are due to R. C. Thompson for making many of the measurements upon which the conclusions drawn herein were based. The assistance of P. C. Reynard and W. E. Thomas, Jr., is also sincerely appreciated.

\section{References}

Alexeff, I., and R. V. Neidigh (1961), Observations of ionic sound waves in plasmas, Phys. Rev. Letters 7, No. 6, 223-225.

Alexeff, I., and R. V. Neidigh (1963), Observations of ionic sound waves in plasmas: their properties and applications, Phys. Rev. 129, No. 2, 516-527.

Jephcott, D. F. (1959), Alfvén waves in a gas discharge, Nature (London) 183, No. 4676, 1652-1654.

Jephcott, D. F. and A. Malein (1964), Experimental study of fast hydromagnetic waves in an argon plasma, Proc. Roy. Soc. (London) Ser. A, 278, No. 1373, 243-255.
Lidsky, L. M., S. D. Rothleder, D. J. Rose, S. Yoshikawa, C. Michelson, and R. J. Mackin, Jr. (1962), Highly ionized hollow cathode discharge, J. Appl. Phys. 33, No. 8, 2490-2497.

McLane, C. K., W. E. Thomas, Jr., and R. C. Thompson (1963), Magnetically stabilized arc, Bull. Am. Phys. Soc. Ser. II, 8, No. 4, 371.

McLane, C. K., S. Takeda, W. E. Thomas, Jr., and R. C. Thompson (1965), Electron density measurements in the magnetically confined arc, to be published in the J. Appl. Phys.

Nagao, S., and T. Sato (1960), Some experiments on plasma Alfvén waves, J. Phys. Soc. Japan 15, No. 4, 735.

Weinstock, J. (1964), Oscillations of a rotating inhomogeneous cylindrical plasma, submitted to Phys. Fluids.

Wilcox, J. M., A. W. DeSilva, and W. S. Cooper, III (1961), Experiments on Alfvén-wave propagation, Phys. Fluids 4, No. 12, 1506-1513.

(Paper 69D3-475) 